\title{
OFDM System Identification for Cognitive Radio Based on Pilot-Induced Cyclostationarity
}

\author{
François-Xavier Socheleau*, Philippe Ciblat** and Sébastien Houcke* \\ *Institut TELECOM; TELECOM Bretagne, \\ Email: \{fx.socheleau, sebastien.houcke\}@telecom-bretagne.eu \\ **Institut TELECOM; TELECOM ParisTech, \\ Email: philippe.ciblat@telecom-paristech.fr
}

\begin{abstract}
In the context of cognitive radio, this paper addresses the challenge of OFDM system identification. We show that it is possible to take advantage of pilot tone structures to perform identification. Pilot subcarriers usage is of great interest since it enables to discriminate OFDM systems that have the same modulation parameters (subcarrier spacing, cyclic prefix duration, etc.). The proposed method relies on the periodic redundancy often induced between pilot symbols. We demonstrate that most OFDM systems (Wifi, WiMAX, DVB-T, etc.) can be identified thanks to this redundancy by conducting a hypothesis test based on second order statistics. Detailed numerical examples demonstrate the efficiency of the proposed identification criterion in various kinds of environments.
\end{abstract}

\section{INTRODUCTION}

$\mathbf{T}$ HE increasing demand of new services constrains wireless systems to move towards a more intelligent control of the limited spectrum resources. [1] laid the foundation of the Cognitive Radio (CR) concept that is now considered as a key paradigm to evolve from the current fixed spectrum usage to opportunistic spectrum sharing models tailored to address the frequency resource scarcity issue. CR promises the deployment of flexible wireless systems able to reconfigure themselves (i.e. adapt their modulation parameters, carrier frequency, power, etc.) with regard to the surrounding radio environment and regulatory policies. Spectrum sensing and especially system identification is therefore a crucial step towards radio environment awareness. In this paper we focus on OFDM based systems as it becomes the physical layer for many wireless standards [2]-[5]. Existing OFDM identification algorithms are mainly based on modulation parameter estimation [6]-[8]. Considering the increasing interest in OFDM by the wireless designers, modulation parameters of such systems are likely to become closer and closer. For instance, 3GPP/LTE [5] and Fixed WiMAX [2] systems already have a subcarrier spacing only different from $4 \%$ which may prevent from getting an accurate system identification based on the subcarrier spacing estimation principle. To overcome this limitation, few methods involving more particular signatures have been suggested in [9]-[11]. These methods either add overhead, and thus reduce systems capacity, or are only dedicated to OFDM systems with specific pilot tone configurations.

In this contribution, we propose a solution to address both issues. By studying existing OFDM systems (Wifi, WiMAX, DVB-T etc.), it can be noticed that pilot symbols, used for channel estimation and/or synchronization purposes, are often replicated with regard to a pre-defined time/frequency distribution. This property induces correlation between pilot subcarriers that can be exploited in conjunction with the periodicity of the time/frequency pilot mapping to perform system identification. In that case, identification can be based upon the knowledge of pilot structures without knowledge of pilot symbols. We hereafter generalize the pilot correlation property and adapt it to the context of cognitive radio under the concept of pilot-induced cyclostationary (PIC) signatures. We show that these signatures can be reliably identified thanks to a hypothesis test based on $2^{\text {nd }}$ order statistics. As in [11], we here suggest to take advantage of signatures created as a side-effect of existing PIC structures to identify standards such as Wifi, WiMAX and DVB-T and also advocate to extend PIC use to future cognitive systems.

The paper is organized as follows: Section II describes the pilot-assisted OFDM system model. Section III recalls the cyclostationarity basis and introduces the pilot induced cyclostationary (PIC) signature scheme with its corresponding identification algorithm. Identification performance is assessed through simulations in Section IV. Finally, conclusions are presented in Section V.

\section{Pilot-ASSisted OFDM System MOdel}

\section{A. Signal model}

Assuming that a transmitted OFDM symbol consists of $N$ subcarriers, the discrete-time baseband equivalent transmit signal is given by

$$
x(m)=\sqrt{\frac{E_{s}}{N}}\left[x_{d}(m)+x_{t}(m)\right],
$$

where

$$
\begin{gathered}
x_{d}(m)=\sum_{k \in \mathbb{Z}} \sum_{\substack{n=0 \\
n \notin \mathcal{I}(k)}}^{N-1} a_{k}(n) e^{2 i \pi \frac{n}{N}(m-D-k(N+D))} \\
. g(m-k(N+D))
\end{gathered}
$$

and

$$
\begin{gathered}
x_{t}(m)=\sum_{k \in \mathbb{Z}} \sum_{n \in \mathcal{I}(k)} b_{k}(n) e^{2 i \pi \frac{n}{N}(m-D-k(N+D))} \\
. g(m-k(N+D)) .
\end{gathered}
$$


$E_{s}$ is the signal power and $a_{k}(n)$ are the transmit data symbols at $n$-th subcarrier of $k$-th OFDM block. These data symbols are assumed to be independent and identically distributed (i.i.d), $D$ is the CP length and $m \mapsto g(m)$ is the pulse shaping filter. $\mathcal{I}(k)$ denotes the set of pilot subcarrier indexes of the $k$-th symbol and $b_{k}(n)$ are the pilot symbols.

Let $\{h(l)\}_{l=0, \cdots, L-1}$ be the baseband equivalent discretetime channel impulse response of length $L$. Unless otherwise stated, the channel is assumed to be time invariant. Notice that impact of channel variations is discussed in Section IV. The received samples of the OFDM signal are then expressed as

$$
y(m)=e^{-i\left(2 \pi \varepsilon \frac{m-\tau}{N}+\theta\right)} \sum_{l=0}^{L-1} h(l) x(m-l-\tau)+\eta(m),
$$

where $\varepsilon$ is the carrier frequency offset (normalized by the subcarrier spacing), $\theta$ the initial arbitrary carrier phase, $\tau$ the timing offset and $\eta(m)$ a zero mean circularly-symmetric complex-valued white Gaussian noise of variance $\sigma^{2}$ per complex dimension. The receiver does not know these parameters $\left(\{h(l)\}_{l=0, \cdots, L-1}, \varepsilon, \theta, \tau, \sigma^{2}\right)$.

\section{B. Pilot tone arrangements}

As depicted in Figure 1, three different types of pilot tone arrangements are usually set up in OFDM systems to meet the channel estimation requirements. The first one is the block type configuration used under the assumption of slow fading channel. Pilot tones are in that case mapped onto all subcarriers of OFDM symbols within a specific period $K$ so that $\mathcal{I}(k)$ verifies

$$
\mathcal{I}(k)= \begin{cases}\{0, \cdots, N-1\}, & \text { if } k=m K(m \in \mathbb{Z}) \\ \emptyset & \text { otherwise. }\end{cases}
$$

The second one, comb-type pilot configuration, is introduced to satisfy the need for equalizing when the channel quickly

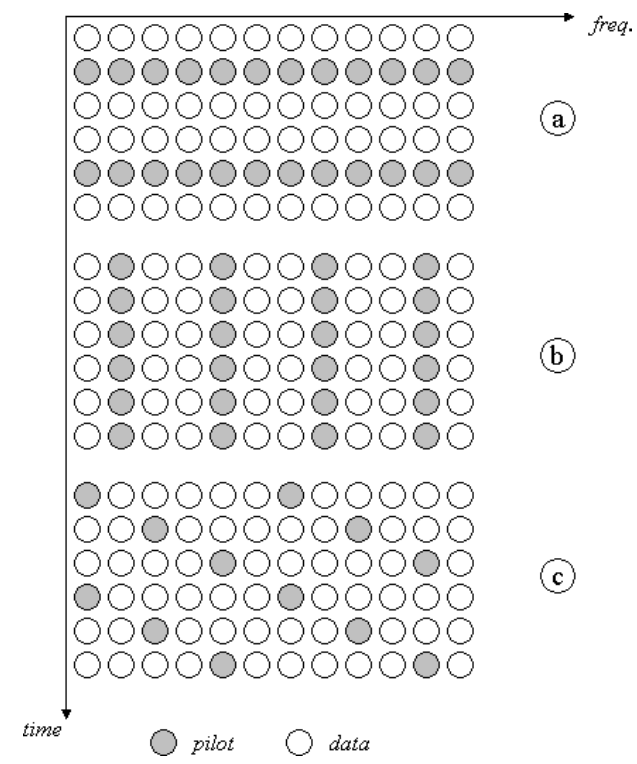

Fig. 1. Examples of pilot tone arrangement: a) block type configuration, b) comb-type configuration, c) circular configuration. varies. Pilot tones are mapped onto certain subcarriers of each OFDM symbol such that $\mathcal{I}(k)=\mathcal{I}$ where $\mathcal{I}$ is any subset of $\{0, \cdots, N-1\} \forall k$. The last arrangement is a circular configuration where the set of pilot subcarrier indexes periodically change such that $\mathcal{I}(k+K)=\mathcal{I}(k)$ where $K \in \mathbb{Z}$ and $\mathcal{I}(k) \neq \emptyset$. Such a scheme is used under the assumption of fast fading channel and presents the interest of avoiding cases where a given pilot subcarrier is attenuated by the channel for a period of several symbols. Note that some OFDM systems (e.g. [4] and [12]) make a joint use of these arrangements.

\section{Pilot-INDUCED CYClOSTATIONARITY (PIC) IDENTIFICATION}

\section{A. Background on cyclostationarity}

Two complex discrete time stochastic processes $x_{k}$ and $y_{k}, k \in \mathbb{Z}$, are said to exhibit joint second-order almost cyclostationarity in the wide sense if the cross correlation function

$$
R_{x y}(k, u) \triangleq \mathbb{E}\left[x_{k} y_{k+u}^{*}\right],
$$

where $*$ stands for complex conjugation, admits series representation

$$
R_{x y}(k, u)=\sum_{\alpha \in \mathcal{A}_{x y}} R_{x y}^{\alpha}(u) e^{i 2 \pi \alpha k}
$$

where

$$
R_{x y}^{\alpha}(u) \triangleq \lim _{M \rightarrow+\infty} \frac{1}{2 M+1} \sum_{k=-M}^{M} R_{x y}(k, u) e^{-i 2 \pi \alpha k}
$$

is the cyclic cross correlation function (CCCF) and

$$
\mathcal{A}_{x y} \triangleq\left\{\alpha \in \left[-1 / 2 ; 1 / 2\left[\mid \exists u \text { s.t. } R_{x y}^{\alpha}(u) \neq 0\right\}\right.\right.
$$

is a countable set of cycle frequencies $\alpha$ [13]. Note that the $\mathrm{CCCF}$ is periodic in $\alpha$ with period 1 .

\section{B. Signature generation}

As illustrated in subsection II-B, the time/frequency pilot tone distribution is always deterministic to meet the channel estimation requirements. As the number of pilot tones is finite, the deterministic characteristic of the pilot tone distribution can be expressed as $\mathcal{I}(k+K)=\mathcal{I}(k), K \in \mathbb{Z}$ for any combination of arrangements described in subsection II-B. For the particular case of comb-type arrangement, note that $K=1$. Such a periodicity is a useful property that can be exploited to induce cyclostationarity in OFDM frames through careful choice of pilot symbols $b_{k}(n)$.

Let $c_{k}(n)$ be the $k$-th symbol on subcarrier $n$ such that

$$
c_{k}(n)= \begin{cases}b_{k}(n), & \text { if } n \in \mathcal{I}(k) \\ a_{k}(n) & \text { otherwise. }\end{cases}
$$

If the pilot tones are designed such that

$$
b_{k}(p)=b_{k+d^{(p, q)}}(q) e^{i \varphi}
$$

with $d^{(p, q)} \in \mathbb{Z}$ and $\varphi \in[-\pi ; \pi[$ then, as shown in appendix A, processes $\left\{c_{k}(p)\right\}_{k}$ and $\left\{c_{k}(q)\right\}_{k}$ are jointly cyclostationary with $\mathcal{A}_{(p, q)} \triangleq \mathcal{A}_{c_{k}(p) c_{k}(q)}=\left\{\frac{m-\lfloor K / 2\rfloor}{K}, m \in\{0, K-1\}\right\}$ where \lfloor\rfloor stands for integer flooring. 
In the framework of OFDM system identification, joint cyclostationary structure is of great interest to generate system specific signatures. Eq. (6) indicates that it is possible to design a given signature $S$ by choosing particular combinations of $p, q, d^{(p, q)}$ and $K$. Such a signature $S$ is then defined as

$$
S \triangleq\left\{\left(p, q, d^{(p, q)}, K\right) \mid \mathcal{A}_{(p, q)} \neq \emptyset\right\} .
$$

As examples, DVB-T, Wifi and WiMAX pilot structures can be seen as (unintentional) PIC signatures where

- DVB-T: $\mathrm{K}=4, d^{(p, q)}=0,1,2$ or $3,(\mathrm{p}, \mathrm{q})$ can be any pilot tone pair.

- Wifi: $\mathrm{K}=1, d^{(p, q)}=0$, (p,q) can be any pilot tone pair.

- WiMAX: see section IV.

Note that the term $e^{i \varphi}$ in Eq. (6) is here to bring flexibility to PIC structures and to prevent coherent addition of pilot symbols which increases the peak-to-average power ratio.

\section{Identification cost function}

Systems satisfying Eq. (1) and (7) have a periodic cross-correlation function $R_{c^{(p, q)}}\left(k, d^{(p, q)}\right)=$ $\mathbb{E}\left[c_{k}(p) c_{k+d^{(p, q)}}^{*}(q)\right]$ and can thus be discriminated by exploiting the cyclic cross-correlation function (CCCF)

$$
\begin{aligned}
R_{c^{(p, q)}}^{\alpha}\left(d^{(p, q)}\right) \triangleq & \lim _{M \rightarrow+\infty} \frac{1}{2 M+1} . \\
& \sum_{k=-M}^{M} \mathbb{E}\left[c_{k}(p) c_{k+d^{(p, q)}}^{*}(q)\right] e^{-i 2 \pi \alpha k} .
\end{aligned}
$$

We propose to perform the system identification on the evaluation of the CCCF energy at cyclic frequencies $\alpha \in \mathcal{A}_{(p, q)}$. In practice the symbols $c_{k}(n)$ are only accessible via the observations $Y_{k}(n)$ expressed as

$$
Y_{k}(n) \triangleq \frac{1}{\sqrt{N}} \sum_{m=0}^{N-1} y[k(N+D)+D+m] e^{-2 i \pi \frac{n m}{N}}
$$

which, in the case of perfect synchronization (i.e. $\varepsilon=0, \tau=0$ and $\theta=0$ ), simplifies to

$$
Y_{k}(n)=H(n) c_{k}(n) \sqrt{E_{s}}+\mathfrak{N}_{k}(n)
$$

where $H(n)$ and $\mathfrak{N}_{k}(n)$ are respectively the channel frequency response at subcarrier $n$ and the noise at subcarrier $n$ of the $k$-th received symbol. Considering the channel frequency response unknown at reception, we therefore have to consider the CCCF of $\left(Y_{k}(p), Y_{k}(q)\right)$ instead of $\left(c_{k}(p), c_{k}(q)\right)$. If we assume that $M$ OFDM symbols are available at reception, the CCCF energy of $y_{k}(p)$ and $y_{k}(q)$ is evaluated thanks to the cost function defined as

$$
J_{P I C} \triangleq \sum_{(p, q) \in \xi}\left(\sum_{\alpha \in \mathcal{A}_{(p, q)}}\left|\hat{R}_{\tilde{Y}^{(p, q)}}^{\alpha}\left(d^{(p, q)}\right)\right|^{2}\right)
$$

where

$$
\begin{aligned}
\hat{R}_{\tilde{Y}^{(p, q)}}^{\alpha}\left(d^{(p, q)}\right)= & \frac{1}{M-d^{(p, q)}} . \\
& \sum_{k=0}^{M-d^{(p, q)}-1} \tilde{Y}_{k}(p) \tilde{Y}_{k+d^{(p, q)}}^{*}(q) e^{-i 2 \pi \alpha k}
\end{aligned}
$$

and $\xi=\left\{(p, q) \mid \mathcal{A}_{(p, q)} \neq \emptyset\right.$ and $\left.d^{(p, q)}+K \leq M\right\}$. Note that in order to get the criterion $J_{P I C}$ less sensitive to the received signal gain, each term $Y_{k}(n)$ in Eq. (9) is normalized so that

$$
\tilde{Y}_{k}(n)=\frac{Y_{k}(n)}{\sqrt{\widehat{\operatorname{Var}}[Y(n)]}},
$$

where $\operatorname{Var}[$.$] denotes the variance and$

$$
\widehat{\operatorname{Var}}[Y(n)]=\frac{1}{M} \sum_{k=0}^{M-1}\left|Y_{k}(n)\right|^{2} .
$$

\section{Decision statistics}

Considering that the system to be identified embeds a PIC signature defined by Eq. (7), our identification problem previously described boils down to a standard detection problem that can be formulated as the following binary hypothesis test

$$
\left\{\begin{aligned}
\mathcal{H}_{0}: \quad & y(m) \text { writes as in Eq. (3) without PIC structure } \\
& \text { or with PIC structure defined as } \\
& S^{\prime}=\left\{\left(p^{\prime}, q^{\prime}, d^{\left(p^{\prime}, q^{\prime}\right)}, K^{\prime}\right) \mid \mathcal{A}^{\prime}{ }_{\left(p^{\prime}, q^{\prime}\right)} \neq \emptyset\right\} \\
& \text { such that } S^{\prime} \neq S . \\
\mathcal{H}_{1}: \quad & y(m) \text { writes as in Eq. (3) with PIC structure } \\
& \text { defined as } S=\left\{\left(p, q, d^{(p, q)}, K\right) \mid \mathcal{A}_{(p, q)} \neq \emptyset\right\}
\end{aligned}\right.
$$

To decide the most likely hypothesis, we propose a detection test constrained by the asymptotic false alarm probability similar to what is suggested in [11]. The decision is made by comparing $J_{P I C}$ to a positive threshold such that

$$
J_{P I C} \underset{\mathcal{H}_{0}}{\stackrel{\mathcal{H}_{1}}{\gtrless}} \Lambda
$$

with $\Lambda$ defined as

$$
\mathcal{F}_{J_{P I C} \mid \mathcal{H}_{0}}(\Lambda)=1-P_{f a}
$$

where $\mathcal{F}_{J_{P I C} \mid \mathcal{H}_{0}}$ is the cumulative distribution function (cdf) of $J_{P I C}$ when $\mathcal{H}_{0}$ holds and $P_{f a}$ is the tolerated false alarm probability. Moreover, we based our decision on the following assumptions:

Assumption 1: We assume that the number of possible PIC signatures are large enough to design OFDM systems satisfying $S^{\prime} \cap S=\emptyset, \forall S^{\prime} \neq S$.

Assumption 2: We assume perfect synchronization at reception (i.e. $\varepsilon=0, \tau=0$ and $\theta=0$ in Eq. (3)). Synchronization impairments are studied in Section III-E.

Assumption 3: Under $\mathcal{H}_{0}$, at a given subcarrier $p$, the symbols $\left\{c_{k}(p)\right\}_{k}$ are assumed to be i.i.d.

The relevance of the hypothesis test given by Eq. (12) can be easily justified by looking at the mean of the estimated CCCF, defined in Eq. (9), under both hypotheses.

$$
\begin{aligned}
\mathbb{E}\left[\hat{R}_{\tilde{Y}(p, q)}^{\alpha}\left(d^{(p, q)}\right)\right] & =R_{\tilde{Y}(p, q)}^{\alpha}\left(d^{(p, q)}\right) \\
& =\left\{\begin{array}{cc}
0 & \text { under } \mathcal{H}_{0} \\
\frac{\mu^{(p, q)}}{K}, \forall \alpha \in \mathcal{A}_{(p, q)} & \text { under } \mathcal{H}_{1}
\end{array}\right.
\end{aligned}
$$

where $\mu^{(p, q)}$ denotes a term proportional to the subcarrier to noise ratio. It is then straightforward to see that the distance between the two asymptotic probability distribution functions 
of $J_{P I C}$ is non null and makes possible the discrimination between the two hypotheses.

In order to find a relevant threshold $\Lambda$ to perform the identification, we hereafter derive the asymptotic statistical behavior of $J_{P I C}$ under $\mathcal{H}_{0}$.

As shown in appendix $\mathrm{B}$, under hypothesis $\mathcal{H}_{0}, J_{P I C}$ is a sum of weighted noncentral chi-square variables with a cumulative distribution function that can be expressed as Laguerre series of the form

$$
\begin{aligned}
\mathcal{F}_{J_{P I C} \mid \mathcal{H}_{0}}(x)= & \frac{e^{-\frac{x}{2 \omega}}}{(2 \omega)^{\zeta+1}} \frac{x^{\zeta}}{\Gamma(\zeta+1)} . \\
& \sum_{k \geq 0} \frac{k ! m_{k}}{(\zeta+1)_{k}} \mathrm{~L}_{k}^{(\zeta)}\left(\frac{(\zeta+1) x}{2 \omega \nu}\right),
\end{aligned}
$$

$\forall \nu>0$ and $\omega>0$, with $\zeta=\sum_{(p, q) \in \xi} \operatorname{card}\left(\mathcal{A}_{(p, q)}\right)$ and $\mathrm{L}_{k}^{(\zeta)}$ the $k$-th generalized Laguerre polynomial verifying

$$
\mathrm{L}_{k}^{(\zeta)}(x) \triangleq \sum_{m=0}^{k}\left(\begin{array}{c}
k+\zeta \\
k-m
\end{array}\right) \frac{(-x)^{m}}{m !} .
$$

$\Gamma(x) \triangleq \int_{0}^{\infty} t^{x-1} e^{-t} d t$ and $(.)_{k}$ denotes the Pochhammer symbol defined as $(x)_{k} \triangleq \frac{\Gamma(x+k)}{\Gamma(x)}$. The coefficients $m_{k}$ satisfy the following recurrent relation

$$
m_{k}=\frac{1}{k} \sum_{j=0}^{k-1} m_{j} g_{k-j}, \quad k \geq 1
$$

with

$$
\begin{aligned}
m_{0}= & 2(\zeta+1)^{\zeta+1} \frac{\omega^{\zeta+1}}{\zeta+1-\nu} . \\
& \prod_{(p, q) \in \xi}\left(\omega \nu+\frac{\zeta+1-\nu}{2\left(M-d^{(p, q)}\right)}\right)^{-\operatorname{card}\left(\mathcal{A}_{(p, q)}\right)}
\end{aligned}
$$

and

$$
\begin{aligned}
g_{j}= & \left(\frac{-\nu}{\zeta+1-\nu}\right)^{j}+ \\
& \sum_{(p, q) \in \xi} \operatorname{card}\left(\mathcal{A}_{(p, q)}\right)\left(\frac{\nu\left(2\left(M-d^{(p, q)}\right) \omega-1\right)}{2\left(M-d^{(p, q)}\right) \omega \nu+\zeta+1-\nu}\right)^{j}, \\
& j \geq 1 .
\end{aligned}
$$

Note that the Laguerre expansion depends on $\nu$ and $\omega$ that can be arbitrarily chosen. These parameter choice only have an impact on the convergence speed and on the uniform convergence property. Moreover, for computer implementation, the Laguerre expansion has to be truncated. The number of terms to consider within the series can be estimated using the truncation error analytical expression given in [14].

\section{E. Effect of synchronization errors}

Timing missynchronization and/or frequency offset deteriorates the observations $Y_{k}(n)$ as inter-symbol (ISI) and intercarrier (ICI) interferences occur. These interferences result in attenuation of the cost function $J_{P I C}$ which has a bad impact on identification performance as it reduces the distance between the distribution of $J_{P I C} \mid \mathcal{H}_{1}$ and $J_{P I C} \mid \mathcal{H}_{0}$. To overcome this performance loss, $\varepsilon$ and $\tau$ can be estimated as

$$
[\hat{\varepsilon}, \hat{\tau}]=\underset{(\varepsilon, \tau)}{\operatorname{argmax}} J_{P I C} .
$$

\section{Simulations}

\section{A. Simulation context}

In the following, all the results are averaged over 1000 Monte Carlo runs. The asymptotic false alarm probability $P_{f a}$ is fixed to 0.01. The Signal-to-Noise Ratio (SNR) is defined as $\operatorname{SNR}(\mathrm{dB})=10 \log _{10}\left(E_{s} / \sigma^{2}\right)$. When notified, the propagation channel simulated is a time-variant discrete-time channel $\left\{h_{k}(l)\right\}_{l=0, \cdots, L}$ with $L=D$ and an exponential decay profile for its non-null component (i.e., $\mathbb{E}\left[\left|h_{k}(l)\right|^{2}\right]=G e^{-l / \beta}$ for $l=0, \cdots, L$ and $G$ is chosen such that $\left.\sum_{l=0}^{L} \mathbb{E}\left[\left|h_{k}(l)\right|^{2}\right]=1\right)$. The channel time variation is simulated using Jake's model.

\section{B. PIC signatures identification performance}

We here simulate a 512-subcarrier OFDM system with a subcarrier mapping scheme equivalent to the Mobile WiMAX DL-PUSC [12] one. We recall that it is designed with 60 pilot, 360 data, 91 guard and $1 \mathrm{DC}$ subcarrier. D is set to $64 . K=$ 2 and joint cyclostationarity is induced on 1770 pilot pairs with $d^{(p, q)}=0$ or 1 . To limit the identification algorithm computational complexity, we test only 30 pairs among all admissible $(p, q)$.

In Figure 2, we plot the correct detection probability versus SNR in the context of AWGN channel for various observation window lengths. We observe that the PIC criterion exhibits excellent performance from $-6 \mathrm{~dB}$ for a number of symbols as small as 25 . The identification rate is significantly improved as the number of available symbols increases. Figure 2 also depicts the impact of different synchronization assumptions on the detection probability. For the simulation, uniformly distributed random $\varepsilon$ and $\tau$ were generated with $-0.5 \leq \varepsilon \leq 0.5$ and $-0.5(N+D) \leq \tau \leq 0.5(N+D)$. On this figure, it can be seen that the loss due to missynchronization can be very high (up to $6 \mathrm{~dB}$ ). This justifies the need for a synchronization method based on Eq. (14) as after correction, this loss is reduced to less than $0.5 \mathrm{~dB}$ for $M=25 . \varepsilon$ and $\tau$ were estimated by maximizing $J_{P I C}$ over a grid with a step of 0.2 over $\varepsilon$ and $0.15(N+D)$ over $\tau$.

In Figure 3, we plot the correct detection probability versus SNR when the frequency-selective channel is time-variant. Various values of maximum Doppler frequencies $f_{d}$ have been inspected. We can see that our algorithm is quite robust to Doppler spread below $100 \mathrm{~Hz}$ and that performance degrades up to $4 \mathrm{~dB}$ for $f_{d}=500 \mathrm{~Hz}$ (at $3 \mathrm{GHz}$, this corresponds to a relative velocity of $180 \mathrm{kph}$ ). However, note that even for $f_{d}=500 \mathrm{~Hz}$, the identification performance is still satisfying in the standard operating SNR range in a mobile environment.

\section{PIC vs m-sequence identification}

We here compare the PIC method with the state of the art pilot structure based identification algorithm described in 


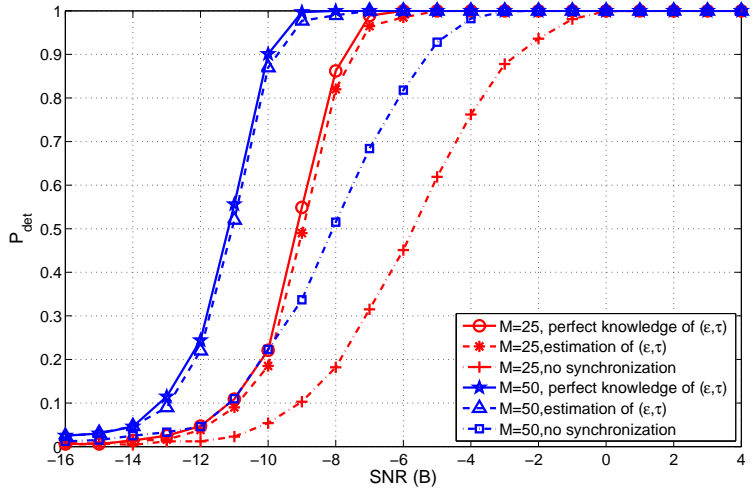

Fig. 2. Effect of SNR and the observation window length as well as synchronization assumptions on the correct detection probability.

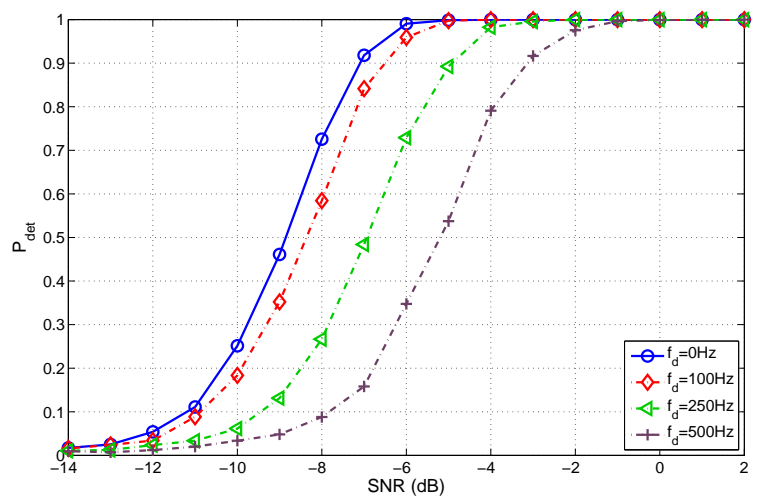

Fig. 3. Effect of Doppler spread on the correct detection probability $(M=$ $25, \beta=0.5 D)$.

[11]. This algorithm relies on the property of $m$-sequences that modulate pilots of systems like [3] and [2]. As shown in [11], fixed WiMAX can be identified thanks to $m$-sequences but can also be identified using the PIC criterion described in this paper. Indeed, from [2], we get $K=1$ and $d^{(p, q)}=0$ with $\xi$ defined as all possible pair of pilot tones (that is $\operatorname{card}(\xi)=28$ ), $\mathrm{D}$ is set to 32 .

Figure 4 compares both methods and clearly indicates that for fixed WiMAX identification, the PIC criterion outperforms (up to $4 \mathrm{~dB}$ ) the $m$-sequence based algorithm.

\section{CONCLUSION}

In this paper, we introduced a new OFDM system identification algorithm to address part of the spectrum sensing challenge inherent in cognitive radio. The method is based on cyclostationary patterns embedded onto pilot tones. This has the main advantage of not adding any system overhead and makes possible the discrimination of systems with similar modulation parameters. Cyclostationary patterns presented in this contribution consider existing pilot constraints so that the method is compatible with most standard requirements. Simulation results proved the efficiency of the proposed identification criterion and showed its robustness to harsh

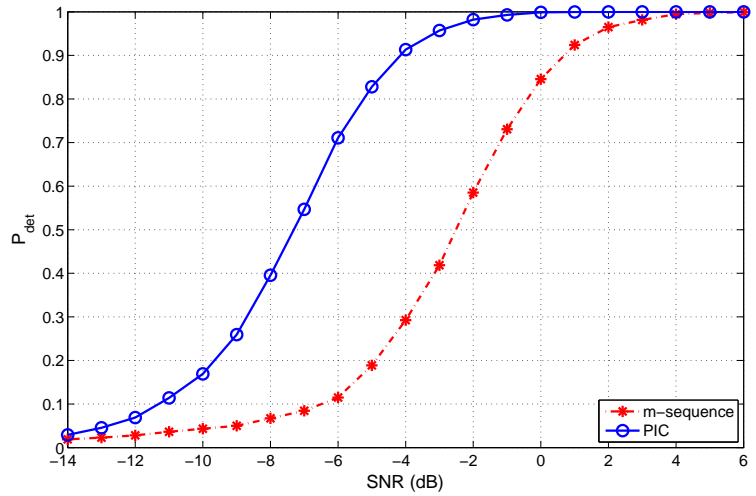

Fig. 4. Identification performance comparison between PIC and $m$-sequence criteria $(M=25, \beta=0.5 D)$.

propagation environment as well as its gain compared with state of the art.

\section{REFERENCES}

[1] J. Mitola III, Cognitive Radio An Integrated Agent Architecture for Software Defined Radio, Ph.D. thesis, KTH Royal Institute of Technology, Stockholm, Sweden, 2000.

[2] IEEE Std. 802.16, Part 16: Air Interface for Fixed Broadband Wireless Access Systems, 2004.

[3] IEEE Std. 802.11a, Part 11: Wireless LAN Medium Access Control (MAC) and Physical Layer (PHY) specifications High-speed Physical Layer in the $5 \mathrm{GHz}$ Band, 1999.

[4] ETSI EN 300744 V1.5.1, Digital Video Broadcasting (DVB); Framing structure, channel coding and modulation for digital terrestrial television, 2004.

[5] 3GPP TR 25.814, Physical Layer Aspects for Evolved UTRA, v.2.0.0, 2006.

[6] T. Yucek and H. Arslan, "OFDM Signal Identification and Transmission Parameter Estimation for Cognitive Radio Applications," in IEEE Glob. Telecom., Nov. 2007, pp. 4056-4060.

[7] A. Bouzegzi, P. Ciblat, and P. Jallon, "Maximum Likelihood based methods for OFDM intercarrier spacing characterization," in IEEE Symp. on Personal, Indoor and Mobile Radio Communications, Sep. 2008.

[8] A. Bouzegzi, P. Jallon, and P. Ciblat, "A fourth-order based algorithm for characterization of OFDM signals," in IEEE Workshop on Signal Processing Advances in Wireless Communications, Jul. 2008.

[9] P.D Sutton, K.E. Nolan, and L.E. Doyle, "Cyclostationary Signatures in Practical Cognitive Radio Applications," IEEE Jounal on selected areas in communications, vol. 26, no. 1, pp. 13-24, Jan. 2008.

[10] K. Maeda, A. Benjebbour, T. Asai, T. Furuno, and T. Ohya, "Recognition Among OFDM-Based Systems Utilizing Cyclostationarity-Inducing Transmission," in IEEE Symp. on New Frontiers in Dynamic Spectrum Access Networks, 2007, pp. 516-523.

[11] F.X Socheleau, S. Houcke, A. Aissa-El-Bey, and P. Ciblat, "OFDM system identification based on $\mathrm{m}$-sequence signatures in cognitive radio context," in IEEE Conf. on Personal, Indoor and Mobile Radio Communications, Sep. 2008.

[12] IEEE Std. 802.16, Part 16: Air Interface for Fixed and Mobile Broadband Wireless Access Systems, Amendment 2: Physical and Medium Access Control Layers for Combined Fixed and Mobile Operations in License Bands and Corrigendum 1, 2005.

[13] W. A. Gardner, A. Napolitano, and L. Paurac, "Cyclostationarity: Half a century of research," Signal processing, vol. 86, no. 4, pp. 639-697, Apr. 2006

[14] A. Castano Martinez and F. Lopez Blazquez, "Distribution of a sum of weighted noncentral chi-square variables," Sociedad de Estadistica e Investigacion Operativa test, vol. 14, no. 02, pp. 397-415, 2005.

[15] P.J. Brockwell and R.A. Davis, Time Series: theory and analysis, Springer, 1991. 


\section{APPENDIX A}

$\left(c_{k}(p), c_{k}(q)\right)$ CCCF DERIVATION

The CCCF of $c_{k}(p)$ and $c_{k}(q)$ is defined as

$R_{c^{(p, q)}}^{\alpha}(u) \triangleq \lim _{M \rightarrow+\infty} \frac{1}{2 M+1} \sum_{k=-M}^{M} \mathbb{E}\left[c_{k}(p) c_{k+u}^{*}(q)\right] e^{-i 2 \pi \alpha k}$.

The data symbols $a_{k}(n)$ being i.i.d and $b_{k}(p)=$ $b_{k+d^{(p, q)}}(q) e^{i \varphi}$, it follows that

$$
\mathbb{E}\left[c_{k}(p) c_{k+d^{(p, q)}}^{*}(q)\right]=\sigma_{b}^{2} e^{-i \varphi} \sum_{m \in \mathbb{Z}} \delta\left[k-m K-k_{0}\right]
$$

where $\delta[$.$] is the Kronecker delta, \sigma_{b}^{2}$ is the variance of symbols $b_{k}(n)$ and $k_{0}$ is the index of the first OFDM symbol embedding pilot tones. Hence,

$$
R_{c^{(p, q)}}^{\alpha}\left(d^{(p, q)}\right)=\frac{\sigma_{b}^{2} e^{-i\left(2 \pi \alpha k_{0}+\varphi\right)}}{K} \sum_{m \in \mathbb{Z}} \delta\left[\alpha-\frac{m}{K}\right] .
$$

Therefore, for $\alpha \in\left[-1 / 2 ; 1 / 2\left[, R_{c^{(p, q)}}^{\alpha}\left(d^{(p, q)}\right) \neq 0\right.\right.$ iff $\alpha \in$ $\left\{\frac{m-\lfloor K / 2\rfloor}{K}, m \in\{0, K-1\}\right\}$.

\section{APPENDIX B}

\section{$J_{P I C} \mid \mathcal{H}_{0}$ CDF DERIVATION}

We first derive the expectation and variance of the $\mathrm{CCCF}$ $\hat{R}_{\tilde{Y}(p, q)}^{\alpha}\left(d^{(p, q)}\right)$ defined in Eq. (9).

The expectation of $\hat{R}_{\tilde{Y}(p, q)}^{\alpha}\left(d^{(p, q)}\right)$ is given by

$$
\begin{aligned}
& \mathbb{E}\left[\hat{R}_{\tilde{Y}^{(p, q)}}^{\alpha}\left(d^{(p, q)}\right)\right] \\
& =\frac{1}{M-d^{(p, q)}} \sum_{k=0}^{M-d^{(p, q)}-1} \mathbb{E}\left[\tilde{Y}_{k}(p) \tilde{Y}_{k+d^{(p, q)}}^{*}(q)\right] e^{-i 2 \pi \alpha k} \\
& =R_{\tilde{Y}^{(p, q)}}^{\alpha}\left(d^{(p, q)}\right)
\end{aligned}
$$

From Assumption $1, \mathbb{E}\left[c_{k}(p) c_{k+d^{(p, q)}}^{*}(q)\right]=0$ so that

$$
\mathbb{E}\left[\hat{R}_{\tilde{Y}(p, q)}^{\alpha}\left(d^{(p, q)}\right)\right]=0 .
$$

To compute the covariance, we introduce the covariance matrix defined as

$$
\mathbf{C}=\mathbb{E}\left[(\mathbf{R}-\mathbb{E}\{\mathbf{R}\})(\mathbf{R}-\mathbb{E}\{\mathbf{R}\})^{H}\right]
$$

where the superscript ${ }^{H}$ stands for transpose conjugate and

$$
\begin{aligned}
\mathbf{R}=[ & \hat{R}_{\tilde{Y}_{0}(p, q)}^{\alpha_{0}}\left(d^{(p, q)}\right), \hat{R}_{\tilde{Y}(p, q)}^{\alpha_{1}}\left(d^{(p, q)}\right), \cdots, \\
& \left.\hat{R}_{\tilde{Y}^{(p, q)}\left(\mathcal{A}_{(p, q)}\right)-1}^{\alpha^{\prime}}\left(d^{(p, q)}\right)\right] .
\end{aligned}
$$

If we now focus on each element $[\mathbf{C}]_{i, j}=$ $\mathbb{E}\left[\hat{R}_{\tilde{Y}(p, q)}^{\alpha_{i}}\left(d^{(p, q)}\right)\left(\hat{R}_{\tilde{Y}(p, q)}^{\alpha_{j}}\left(d^{(p, q)}\right)\right)^{*}\right]$, we have:

$$
\begin{aligned}
{[\mathbf{C}]_{i, j}=} & \frac{1}{\left(M-d^{(p, q)}\right)^{2}} . \\
& \sum_{k_{i}, k_{j}=0}^{M-d^{(p, q)}-1} \mathbb{E}\left[\tilde{Y}_{k_{i}}(p) \tilde{Y}_{k_{i}+d^{(p, q)}}^{*}(q) \tilde{Y}_{k_{j}}^{*}(p) \tilde{Y}_{k_{j}+d^{(p, q)}}(q)\right] \\
& . e^{-i 2 \pi\left(k_{i} \alpha_{i}-k_{j} \alpha_{j}\right)} .
\end{aligned}
$$

Once again due to Assumption 1 and due to the circularity of symbols (PSK or QAM), we get

$$
\begin{aligned}
& \mathbb{E}\left[\tilde{Y}_{k_{i}}(p) \tilde{Y}_{k_{i}+d^{(p, q)}}^{*}(q) \tilde{Y}_{k_{j}}^{*}(p) \tilde{Y}_{k_{j}+d^{(p, q)}}(q)\right] \\
& =\mathbb{E}\left[\tilde{Y}_{k_{i}}(p) \tilde{Y}_{k_{j}}^{*}(p)\right] \mathbb{E}\left[\tilde{Y}_{k_{i}+d^{(p, q)}}^{*}(q) \tilde{Y}_{k_{j}+d^{(p, q)}}(q)\right] .
\end{aligned}
$$

From Assumption 3, this term is different from zero only if $k_{i}=k_{j}$. Moreover, as shown in Eq. (10), $\tilde{Y}_{k}(n)$ is expressed as a ratio of two random variables. The variance estimator introduced in Eq. (11) being consistent, it converges almost surely to a constant denoted $v_{n}$ so that, thanks to the asymptotic theory developed in [15], $\tilde{Y}_{k}(n)$ converges in distribution to $Y(n) / \sqrt{v_{n}}$. Thus, for $k_{i}=k_{j}$ and a time invariant propagation channel

$$
\begin{aligned}
& \mathbb{E}\left[\tilde{Y}_{k_{i}}(p) \tilde{Y}_{k_{i}+d^{(p, q)}}^{*}(q) \tilde{Y}_{k_{j}}^{*}(p) \tilde{Y}_{k_{j}+d^{(p, q)}}(q)\right] \\
& =\frac{\left(|H(p)|^{2} \rho(p)+\frac{\sigma^{2}}{N}\right)\left(|H(q)|^{2} \rho(q)+\frac{\sigma^{2}}{N}\right)}{v_{p} v_{q}} \\
& =1
\end{aligned}
$$

as $v_{n}=|H(n)|^{2} \rho(n)+\sigma^{2} / N$ with $\rho(n)$ the signal power of subcarrier $n$. Therefore, the asymptotic covariance is expressed as

$$
\begin{aligned}
& {[\mathbf{C}]_{i, j}=\frac{1}{\left(M-d^{(p, q)}\right)^{2}} \sum_{k=0}^{M-d^{(p, q)}-1} e^{-i 2 \pi k\left(\alpha_{i}-\alpha_{j}\right)}} \\
& =\frac{\sin \left(\pi\left(M-d^{(p, q)}\right)\left(\alpha_{i}-\alpha_{j}\right)\right)}{\left(M-d^{(p, q)}\right)^{2} \sin \left(\pi\left(\alpha_{i}-\alpha_{j}\right)\right)} e^{-i \pi\left(\alpha_{i}-\alpha_{j}\right)\left(M-d^{(p, q)}-1\right)} .
\end{aligned}
$$

From Eq. (16) we can deduce that the correlation between $\hat{R}_{\tilde{Y}(p, q)}^{\alpha_{i}}\left(d^{(p, q)}\right)$ and $\hat{R}_{\tilde{Y}(p, q)}^{\alpha_{j}}\left(d^{(p, q)}\right)$ for $\alpha_{i} \neq \alpha_{j}$ is upper bounded by

$$
\begin{aligned}
\left|[\mathbf{C}]_{i, j}\right| & \leq \frac{1}{\left(M-d^{(p, q)}\right)^{2} \sin \left(\pi\left(\alpha_{i}-\alpha_{j}\right)\right)} \\
& \leq \frac{1}{\left(M-d^{(p, q)}\right)^{2} \sin (\pi / K)} .
\end{aligned}
$$

which implies that as long as $K \ll \pi\left(M-d^{(p, q)}\right)^{2}$, the cyclic cross-correlation coefficients are asymptotically mutually independent. Thus, thanks to the central limit theorem and according to Eqs. (15) and (16)

$$
\hat{R}_{\tilde{Y}(p, q)}^{\alpha}\left(d^{(p, q)}\right) \mid \mathcal{H}_{0} \stackrel{\mathcal{D}}{\longmapsto} \mathcal{C N}\left(0, \frac{1}{M-d^{(p, q)}}\right)
$$

where $\stackrel{\mathcal{D}}{\longmapsto}$ indicates the convergence in distribution. The cyclic cross-correlation coefficients estimate being asymptotic uncorrelated Gaussian variables, it follows that $\tilde{Y} \mid \mathcal{H}_{0}$ can be expressed as a sum of weighted central chi-square variables. Therefore, the characteristic function of $J_{P I C} \mid \mathcal{H}_{0}$ is expressed as

$$
\psi_{J_{P I C} \mid \mathcal{H}_{0}}(t)=\prod_{(p, q) \in \xi}\left(1-\frac{i t}{M-d^{(p, q)}}\right)^{\operatorname{card}\left(\mathcal{A}_{(p, q)}\right)} .
$$

The inversion of this characteristic function using the results presented in [14] concludes the proof. 